\title{
Decreased brain volumes in infants with prenatal opioid exposure
}

Jonathan Dietrich ${ }^{1}$, Zoe Guckien ${ }^{1}$, MaKayla Picklesimer², Christina M Sparks ${ }^{3}$, David M Haas ${ }^{4}$, Senthilkumar Sadhasivam ${ }^{3}$, Rupa Radhakrishnan ${ }^{2}$

${ }^{1}$ Indiana University School of Medicine; ${ }^{2}$ Indiana University School of Medicine, Department of Radiology and Imaging Sciences; ${ }^{3}$ Indiana University School of Medicine, Department of Anesthesia; ${ }^{4}$ Indiana University School of Medicine, Department of Obstetrics and Gynecology

Background/Objective: Previous small studies have shown that prenatal opioid-exposed $(\mathrm{POE})$ infants and older children display decreased cerebral, cerebellar, or subcortical brain volumes. However, these studies are plagued by suboptimal reference standards or were unable to correct for the influence of other environmental factors in older children. Therefore, our goal was to study differences in brain volume of POE infants when compared to a geographically matched population. We hypothesized that there will be a significant decrease in total brain volume of the POE infants in comparison to the non-opioid exposed control infants, including a reduction in the cerebellar volume.

Methods: This was an IRB approved prospective study of mothers and infants with POE and controls without POE. All recruited infants underwent MRI scans of the brain before they reached a corrected age of 2 months. The T1-weighted MRI images were analyzed by Infant FreeSurfer and segmented into ROIs. The segmentations were manually checked and edited. An ANOVA analysis was performed to compare the cerebellar and total brain volume datasets. We corrected for gender, corrected gestational age at MRI scan, and total brain volume where necessary.

Results: 42 infants were included in the study, 21 with POE and 21 control infants. There was a significant difference in the mean gestational age of POE infants (38.28 \pm 2.13$)$ compared to control infants $(39.42 \pm 0.72)$. On quantitative analysis, the POE group had a significantly reduced total brain and supratentorial volume in comparison to the controls. The cerebellar volume was also significantly smaller in POE, but this significance did not persist when the total brain volume was included in the model.

Conclusion: The supratentorial region is affected disproportionately more than the cerebellum in POE. Specific reductions in cortical, subcortical, and white matter volume need to be further investigated and their influence on developmental outcomes need to be studied. 